NBER WORKING PAPER SERIES

\title{
REGIME-SWITCHING BEHAVIOR OF THE TERM STRUCTURE OF FORWARD MARKETS
}

\author{
Elena Tchernykh \\ William H. Branson \\ Working Paper 11517 \\ http://www.nber.org/papers/w11517
NATIONAL BUREAU OF ECONOMIC RESEARCH
1050 Massachusetts Avenue
Cambridge, MA 02138
July 2005

We thank Jean-Michel Bismut, Alex Pomorin, Chris Sims, Mark Taylor, Mark Watson, and participants at seminars at the Central Bank of Brazil, City University of Hong Kong, Hong Kong Institute for Monetary Research and Hong Kong University of Science and Technology for helpful suggestions. The views expressed herein are those of the author(s) and do not necessarily reflect the views of the National Bureau of Economic Research.

(C2005 by Elena Tchernykh and William H. Branson. All rights reserved. Short sections of text, not to exceed two paragraphs, may be quoted without explicit permission provided that full credit, including (C) notice, is given to the source. 
Regime-Switching Behavior of the Term Structure of Forward Markets

Elena Tchernykh and William H. Branson

NBER Working Paper No. 11517

July 2005

JEL No. F31, C13

\section{$\underline{\text { ABSTRACT }}$}

This paper presents techniques for modelling and estimating the behavior of financial market price or return differentials that follow non-linear regime-switching behaviour. The methodology to be used here is estimation of variants of threshold autoregression (TAR) models. In the basic model the differentials are random within a band defined by transactions costs and contract risk; they occasionally jump outside the band, and then follow an autoregressive path back towards the band. The principal reference is Tchernykh (1998). The application here is to deviations from covered interest parity (CIP) between forward foreign exchange (FX) markets in Hong Kong and the Philippines. We have observed that these deviations from the band follow irregular steps, rather than single jumps. Therefore a Modified TAR model (MTAR) that allows for this behaviour is also estimated. The estimation methodology is a regime-switching maximum likelihood procedure. The estimates can provide indicators for policy-makers of the market's expectation of crisis, and could also provide indicators for the private sector of convergence of deviations to their usual bands. The TAR model has the potential to be applied to differentials between linked pairs of financial market prices more generally.

Elena Tchernykh

Moscow Power Institute

Kafedra Vysshej Matematiki

Krasnokazarmennaya Ul, d. 14

Moscow

RUSSIA

etchernykh@aol.com
William H. Branson

Department of Economics

Princeton University

Princeton, NJ 08544

and NBER

wbranson@princeton.edu 


\title{
Regime-switching Behavior of the Term Structure of Forward Markets
}

\author{
Elena Tchernykh and William H. Branson
}

\section{Introduction}

The topic of this paper is modelling and estimating the time-series behavior of deviations from covered interest parity (CIP) in spot and forward FX markets. We study both spot-forward and forward-forward deviations. The latter are deviations from CIP between different forward maturities. The analysis can give us a view of deviations from CIP along the term structure of forwards.

The literature on CIP deviations goes back at least to Keynes in the 1920s and Paul Einzig in the 1930s and 60s. They intuitively described deviations from CIP as random within a neutral band of returns defined by transactions costs and perhaps default risk. Keynes speculated that the size of this band might be 50 basis points for sterling. Later work by Branson (1969) and by Frenkel and Levich (1975) estimated that this band might be as narrow as 25 basis points. Keynes and Einzig also described CIP deviations as occasionally breaking out of the band, and then regressing back toward the band over time.

Deviations from the band present the market with an arbitrage opportunity, a profitable transaction covered against forward exchange risk. This arbitrage is "riskless" in the absence of default risk. Acting on this opportunity, the market drives the deviation back toward the band. This movement may not be instantaneous and complete due to heterogeneity both of information and default expectations. Thus the deviation would regress toward the band over a period of time. This is the regression mentioned by Keynes and Einzig, and it is the main subject of estimation here.

This time-series behavior can be formally modelled as a threshold autoregression process (TAR). The time series in question, here CIP deviations, is stochastic within the neutral band defined by the thresholds, but autoregressive outside them. The band could be asymmetric, if default risk differs depending on the direction of the transactions. The speed of regression could also differ above and 
below the band. Asymmetric bands were estimated in the US - Russian market by Taylor and Tchernykh (2004), these are also evident in the time series data for Brazil and Southeast Asian countries, and are observed here for Hong Kong and the Philippines. The TAR model for time series originated with Howell Tong in 1978, and has been applied to spot-forward CIP deviations more recently by Mark Taylor and co-authors.

In this paper, variants of TAR models are specified and estimated for forwardforward deviations from CIP for Hong Kong and the Philippines. We focus on 3-6 month deviations. Tchernykh has already studied Russia [Tchernykh (1998), Taylor and Tchernykh (2004)] and Brazil [Tchernykh (2002a)], and we are now further studying recent Asia data.

In addition to tranquil periods of normal functioning of the forward markets, the Asian data as well as the data studied here include the relatively turbulent period of the Asian crisis. For a macroeconomic overview of the causes of the crisis see, for example, Corsetti, Pesenti, and Roubini (1999). During a crisis, new information enters the markets sporadically as the authorities are seen to intervene and market expectations become more volatile. As the CIP differentials widen, market participants' estimates of probabilities of default rise. Market participants understand they are in a crisis, and this further increases the volatility of deviations. Thus during the crisis period the thresholds of the TAR model may become econometrically insignificant. We see this result in the data here.

Deviations from CIP, and estimates of the TAR model, can be useful to policy-makers and traders. Deviations tend to signal coming crises. This was clear in the Russian data, and also appears in the Asian data. The TAR model estimates provide measures of significant deviations from CIP before crises. These can be used by policy-makers as signals of bubbles and coming crises. They can be used by traders as the basis for trading rules for arbitrage.

The paper is structured as follows. First, we specify spot-forward and forwardforward deviations. Then we formulate the TAR model and estimators for the TAR model. Next we formulate a modification of the TAR model (MTAR) which includes the Poisson process as well as autoregression outside of the band. Finally, we present the empirical results for the Hong Kong dollar and the Philippine peso. 


\section{Spot-Forward CIP}

The covered interest parity (CIP) theorem for foreign exchange states that the foreign exchange forward premium equals the interest rate differential between two relevant currencies:

$$
\frac{F_{i}}{S}=\frac{\left(1+R_{i}\right)}{\left(1+R_{i}^{*}\right)} \text {. }
$$

Here $F_{i}$ represents the i-period forward exchange rate in terms of units of home currency per unit of foreign exchange, $\mathrm{S}$ is the spot exchange rate, $R_{i}$ is the domestic i-period interest rate, and $R_{i}^{*}$ is the foreign i-period interest rate. In log-linear form the formula for CIP may be expressed as:

$$
\mathrm{f}_{\mathrm{i}}-\mathrm{s}=\mathrm{r}_{\mathrm{i}}-\mathrm{r}{ }_{\mathrm{i}}
$$

If (2) does not hold, then it must be possible to arbitrage risklessly and profitably from one of the currencies into the other. This arbitrage will ensure that equality (2) holds. Equations (1) and (2) are "no arbitrage" conditions, as in Arbitrage Pricing Theory (APT) more generally. Since the forward transaction eliminates any foreign exchange risk exposure, any deviation from covered interest parity must either represent market inefficiency or else a premium arising from perceived risk of default.

\section{Forward -Forward CIP: Extension of the Basic CIP Theory}

A more general form of covered interest arbitrage would involve arbitraging along the term structure of forward differentials. For example, see Taylor and Tchernykh (2004). For no arbitrage to be profitable, the following generalized CIP condition must hold: 


$$
\frac{F_{j}}{F_{i}}=\frac{\left(1+R_{i j}\right)}{\left(1+R_{i j}{ }^{*}\right)} .
$$

Here $F_{i}$ is the forward exchange rate for period $\mathrm{i}$, and similarly $F_{j} ; R_{i j}$ is the

domestic forward interest rate, and $R_{i j}{ }^{*}$ is the foreign forward interest rate between times $\mathrm{i}$ and $\mathrm{j}, \mathrm{i}<\mathrm{j}$. This CIP relationship can also be approximated in log-linear form as

$$
f_{j}-f_{i}=r_{i j}-r *_{i j}
$$

From equation (4), the generalized CIP condition, define the deviation from CIP for arbitrage between maturities $\mathrm{i}$ and $\mathrm{j}, y_{t}$ as

$$
y_{t}=\left(f_{j}-f_{i}\right)-\left(r_{i j}-r_{i j}^{*}\right) \text {. }
$$

Our empirical methodology involves estimating nonlinear time series models for $y_{t}$. In particular, we wish to estimate the boundaries of the neutral band within which arbitrage does not take place, and the speed of mean reversion of deviations from CIP outside of the neutral band.

\section{Threshold Autoregression (TAR) Model}

The basic idea of the threshold model is a local approximation of differing regimes over states, i.e. introduction of regimes via thresholds. The threshold principle allows the analysis of a complex stochastic system by decomposing it into simpler subsystems. Under the threshold principle, we may group a number of finite parametric non-linear time series models - in our case, stochastic behaviour between the thresholds and autoregressive outside the band - into one model to be estimated.

As discussed above, a number of authors have suggested that time series for CIP deviations may be characterised by threshold effects, such that arbitrage occurs mainly once the size of the deviation has passed a certain level. This would suggest that CIP deviations would be largely random in a certain neighbourhood, while deviations from 
CIP outside of this range would not be immediately returned into the neutral band but would instead show a statistical tendency to revert towards the band.

The gradual return to the band could be based on at least two factors. First, arbitrage between forward markets is less well established than spot-forward arbitrage. So information here is likely to be particularly costly and imperfect, as shown in general by Grossman and Stiglitz (1980). This would make information heterogeneous across market participants. Second, the size of transactions needed for profitable arbitrage is likely to be large. This could create differences in liquidity across participants. Therefore, arbitrage will eliminate deviations only gradually.

A parametric model which may capture this non-linear behaviour - and which nests both instantaneous and slower mean-reversion towards the band - is the threshold autoregressive (TAR) model (Tong, 1978, 1983; Granger and Teräsvirta, 1993), which can be extended following the work of Taylor and Peel (1998) to allow for asymmetry in the neutral band. A TAR model for deviations from covered interest parity, $y_{t}$, can be written:

$$
\begin{array}{ll}
y_{t+1}=y_{t}+\varepsilon_{1, t+1} & \text { if } y_{t}<\kappa_{1} \text { and } y_{t}>\kappa_{2} \\
y_{t+1}=\kappa_{1}\left(1-\beta_{1}\right)+\beta_{1} y_{t}+\varepsilon_{2, t+1} & \text { if } y_{t} \geq \kappa_{1}, \\
y_{t+1}=\kappa_{2}\left(1-\beta_{2}\right)+\beta_{2} y_{t}+\varepsilon_{2, t+1} & \text { if } y_{t} \leq \kappa_{2},
\end{array}
$$

where $\varepsilon_{i t} \sim N\left(0, \sigma_{i}^{2}\right), \mathrm{i}=1,2$. Here $\left(\kappa_{2}, \kappa_{1}\right)$ are the upper and lower thresholds of the band, and $\beta_{i} \in(0,1)$ are the autoregressive parameters. The speed of mean-reversion is determined by $\beta_{i}$.

The model of equations (6) can be estimated using a grid search over $\left(\kappa_{2}, \kappa_{1}\right)$. This procedure will yield estimates of the parameters $\left(\kappa_{2}, \kappa_{1}\right)$ that maximize the loglikelihood function:

$$
\begin{gathered}
L\left(\beta_{1}, \beta_{2}, \sigma_{1}^{2}, \sigma_{2}^{2}, \kappa_{1}, \kappa_{2}\right)= \\
-\frac{1}{2} \sum_{y_{t} \in\left(\kappa_{2}, \kappa_{1}\right)}\left[\ln \left(\sigma_{1}^{2}\right)+\varepsilon_{1, t+1}^{2} / \sigma_{1}^{2}\right]-\frac{1}{2} \sum_{y_{t} \notin\left(\kappa_{2}, \kappa_{1}\right)}\left[\ln \left(\sigma_{2}^{2}\right)+\varepsilon_{2, t+1}^{2} / \sigma_{2}^{2}\right] .
\end{gathered}
$$


If there are not enough observations with deviations below the lower threshold, we estimate the upper threshold as follows:

$$
\begin{array}{ll}
y_{t+1}=y_{t}+\varepsilon_{1, t+1} & \text { if } y_{t}<\kappa_{1}, \\
y_{t+1}=\kappa_{1}(1-\beta)+\beta y_{t}+\varepsilon_{2, t+1} & \text { if } y_{t} \geq \kappa_{1},
\end{array}
$$

where $\varepsilon_{i, t+1} \sim N\left(0, \sigma_{i}^{2}\right) \mathrm{i}=1,2$, and $\beta \in(0,1)$. The likelihood function then takes the form:

$$
\begin{gathered}
L\left(\beta, \sigma_{1}^{2}, \sigma_{2}^{2}, \kappa_{1}\right)= \\
-\frac{1}{2} \sum_{y_{t}<\kappa_{1}}\left[\ln \left(\sigma_{1}^{2}\right)+\varepsilon_{1, t+1}^{2} / \sigma_{1}^{2}\right]-\frac{1}{2} \sum_{y_{t} \geq \kappa_{1}}\left[\ln \left(\sigma_{2}^{2}\right)+\varepsilon_{2, t+1}^{2} / \sigma_{2}^{2}\right],
\end{gathered}
$$

and estimation may proceed as before. Estimation of model (6) or (8) using the likelihood function (7) or (9) yields the maximum-likelihood estimates of the parameters $\beta, \sigma_{1}^{2}, \sigma_{2}^{2}$, and $\kappa$.

\section{Modified TAR (MTAR) Model}

The data from Asia shown in Tchernykh (2004), and for the Philippines below, show that deviations from CIP frequently occur in irregular steps, not single jumps as assumed by the classical TAR model. The number and magnitude of these steps differ substantially across episodes. Including these data points in estimation of the classical TAR model would put an upward bias in the estimates of the $\beta$ parameter for the speed of autoregression. These movements reflect a jump process involving information lags.

We will proceed to estimate a Modified TAR model, the MTAR model, to identify the point at which the autoregression takes hold, and then to re-estimate the autoregression. This modification uses the data points in the jumps in the deviations in identifying that point. Consider the state where $y_{t} \geq \kappa_{1}$. Let us define the starting $a_{n}$ and ending $b_{n}$ points for each period $n(n=1, \ldots, N)$ of movement of $y_{t}$ above $k_{1}$ for any $y_{t}$ from the state $y_{t} \geq \kappa_{1}$, and $a_{n}<t<b_{n}$. Than for each interval $\left[a_{n}, b_{n}\right]$, 
$t_{n} \in\left[a_{n}, b_{n}\right]$, we can find a maximum $t_{n, \max }$, such that $y_{t_{n, \max }}=\max _{\left[a_{n}, b_{n}\right]}\left(y_{t}\right)$, $a_{n}<t_{n, \max }<b_{n}, y_{a_{n}}=y_{b_{n}}=k_{1}$.

We define the domain of the deviations outside of the thresholds as

$$
\Omega=\left\{\bigcup_{n=1}^{N}\left[a_{n}, b_{n}\right]\right\},
$$

the domain of the jump process as

$$
\mathrm{P}=\left\{\bigcup_{n=1}^{N}\left[a_{n}, t_{n, \max }\right]\right\},
$$

and the domain of the autoregressive process as

$$
\mathrm{A}=\left\{\bigcup_{n=1}^{N}\left[t_{n, \max }, b_{n}\right]\right\} .
$$

The asymmetric MTAR model can be written as follows.

$$
\begin{array}{ll}
y_{t+1}=y_{t}+\varepsilon_{1, t+1} & \text { if } y_{t}<\kappa_{1}, \\
y_{t+1}=\kappa_{1}(1-\beta)+\beta y_{t}+\varepsilon_{2, t+1} & \text { if } t \in \mathrm{A} .
\end{array}
$$

The likelihood function for the asymmetric MTAR model would be:

$$
\begin{gathered}
L\left(\beta, \sigma_{1}^{2}, \sigma_{2}^{2}, \kappa_{1}\right)= \\
-\frac{1}{2} \sum_{y_{t}<\kappa_{1}}\left[\ln \left(\sigma_{1}^{2}\right)+\varepsilon_{1, t+1}^{2} / \sigma_{1}^{2}\right]-\frac{1}{2} \sum_{y_{t} \geq \kappa_{1}, t \in \Omega} I(t)\left[\ln \left(\sigma_{2}^{2}\right)+\varepsilon_{2, t+1}^{2} / \sigma_{2}^{2}\right],
\end{gathered}
$$

where

$$
I(t)=\left\{\begin{array}{l}
1, \text { if } t \in \mathrm{A} \\
0, \text { if } t \in \mathrm{P}
\end{array} .\right.
$$


Given the model (13.1), (13.2) and the likelihood function (14), estimation can proceed as described earlier. However, the likelihood function (14) and the resulting estimates are conditional on identification of the domain $\mathrm{P}$ of the jump process (11). Our interest here is the estimation of the regression parameter $\beta$ and the associated times of mean reversion. Re-estimation of the MTAR will not affect the parameter estimates conditional on the identification of the domain of the jump process.

Below we present the estimates of the MTAR and TAR models for Hong Kong and the MTAR model for the Philippines. The TAR estimates for the Philippines were presented earlier in Tchernykh (2002b). In the application to the data for the Hong Kong 3-6 month CIP differentials we compare results of the classical TAR and global AR using Monte Carlo likelihood ratio tests to confirm the significance of the estimates, as described below. We then compare the MTAR and TAR estimates of the autoregression parameters.

The maximum-likelihood estimates of the TAR parameters can be tested against the null hypothesis of simple $A R(1)$ mean reversion or zero bandwidth, $\kappa_{1}=\kappa_{2}$, by estimating the restricted $\mathrm{AR}(1)$ model and applying a likelihood ratio test. In this case, the test likelihood ratio statistic is twice the difference between the value of the likelihood function for the $\operatorname{AR}(1)$ model and the maximized value of the likelihood function for the TAR model.

Under the null hypothesis, the thresholds are not identified, so the likelihood ratio statistic will not follow a standard $\chi^{2}$. Therefore, we estimated the empirical marginal significance level of the likelihood ratio statistics through Monte Carlo simulation following a standard procedure. The restricted AR(1) model was estimated using the actual data. The resulting AR(1) parameter estimates were used to calibrate an artificial AR(1) data generating process with Gaussian errors. Five thousand artificial data sets equal in length to the actual data set were generated using this process. The TAR and the AR(1) models were estimated, and the resulting 5000 values of the likelihood ratio statistic were taken as the empirical distribution of the statistic under the null hypothesis.

In the empirical results reported below, if the maximized likelihood statistic is significant at the 95 percent confidence level, the null of AR(1) is rejected and the TAR estimates are said to be confirmed. If the maximized statistic is not significant, the AR(1) 
null is not rejected and the estimates are not confirmed. In either case, the autoregression parameters are re-estimated using MTAR.

\section{Empirical Results: Hong Kong and the Philippines}

In this section we present estimates of MTAR and TAR models for deviations from CIP in the 3 - 6 month forward markets for Hong Kong and the Philippines on daily data for the period 1994-2002. The Hong Kong markets are more developed and stable than those in the Philippines, so estimation on these two can provide a test of the applicability of the models across levels of institutional development. This time period also spans the period of the Asian currency crises. Thus it permits us to examine the behaviour of the data and the applicability of the models across tranquil and crisis periods. These periods are evident in the data shown in Graphs 1 and 5.

We expect variants of the TAR model with well-estimated thresholds to hold during tranquil periods, but not during crises. During a tranquil period the flow of information to the markets and market participants' estimates of the probabilities of default are likely to be relatively homogeneous, thus we expect deviations to be mainly within the neutral band. Occasionally an unanticipated event will send the deviation outside the band, and arbitrage will bring it back. During these episodes we can observe regime-switching behaviour, and it should be possible to estimate a TAR or MTAR model with results that are significant and economically meaningful. These would be confirmed by the Monte Carlo likelihood test.

During a crisis period the flow of information is likely to become irregular, as the market adjusts expectations to rapidly and substantially changing events. These could include unusually large or unanticipated changes in interest rates or exchange market intervention by the authorities. Market participants could begin to base their expectations on their own perceptions of other participants' expectations. In this case, estimates of the probabilities of default could also begin to fluctuate widely. During these periods we expect to see wide and random fluctuations in CIP deviations. In this case, the thresholds of the TAR model disappear, and the estimation results should become insignificant. These would be not confirmed by the Monte Carlo test. We see these differences in the estimates presented here. 
As the markets approach a crisis, we expect that they enter a state of transition from TAR behaviour to crisis behaviour. During such a transition, deviations are likely to grow, and TAR behaviour is likely to weaken. We also see this transition in the data here, and earlier in Tchernykh (2002b) for a wider range of Asian countries.

\section{Hong Kong}

The data on the 3-month - 6-month CIP deviations, defined in equation (5) above, for the Hong Kong dollar - US dollar rates are shown in Graph 1. These are daily data for the period January 1994 through September 2002, taken from Bloomberg. The data have been matched for gaps and differing holidays to provide a full and consistent data set. In the chart a positive deviation means the 6-month forward exceeded the 3month by more than the corresponding interest differential. This implies that arbitrage could be profitable buying 3-month forward and selling 6 month. A negative differential would imply the opposite arbitrage; thus large differentials indicate failures of arbitrage to maintain CIP.

The Hong Kong data of Graph 1 can be divided into three distinct periods, as suggested above. These are shown in Graphs 2, 3, and 4. The period from January 1994 to July 1997 seems to exhibit TAR behavior with an occasional downward spike. For example, in early 1995 the differential jumped to nearly 0.005 , and then reverted to what appears to be a normal range of fluctuation. The data up to July 1997 show occasional short periods of noise and longer periods of what seems to be TAR behavior.

The second period runs from July 1997 to April 2000. With the beginning of the Asian crisis, deviations grew distinctly larger. From late 1997 to late 1998, they remained in the range $0.005-0.025$. Both the mean and the variance of the data increased sharply during the crisis period.

The last period runs from March 2000 to August 2002, after the crisis. This is a tranquil period. We do see below that estimates of a TAR model are confirmed for this period. For each of these three periods, we also estimate the MTAR model, and compare the results with a TAR model. Estimation was performed using several different algorithms. The Broyden, Fletcher, Goldfarb and Shanno (BFGS) algorithm was most stable in terms of convergence, so it is used in all the estimates presented here. We begin by presenting the results from estimation of MTAR for each period.

The estimation results are presented in a standard format for Hong Kong and later for the Philippines. Each graph has two panels. The first panel displays the 
movements of the deviations for the period. The second panel for Hong Kong gives a summary of the MTAR and the original TAR estimates for behaviour above the estimated threshold. The second panel for the Philippines shows only the MTAR estimates. The original TAR estimates for the Philippines are presented in Tchernykh (2002b). The tables in the middle panels show the estimated upper threshold for deviations and the estimated value of autoregressive parameter $\beta$. The TAR and MTAR models both assume that the autoregression coefficient $\beta$ will be between 0 and 1 . Therefore, two t-statistics are presented for $\beta$. The first, t-stat $(0)$, is the usual test of $\beta>0$. The t-stat(1) is the test of $\beta<1$. This is constructed as $(1-\beta) / S E(\beta)$. We will use both t-statistics in evaluating the $\beta$ estimates. Both of these are significant at the 95 percent confidence level in all of the estimates reported here.

The results for the first period in the Hong Kong data are shown in Graph 2. The data in the top panel seem to show TAR behaviour. The results in the second panel first show the estimation of only an upper threshold, here 0.0007 . This means that much of the data before middle 1994 and after January 1995 are above the threshold. The MTAR estimate of $\beta$ is 0.879 , with a t-statistic for the comparison to zero of 31.8 . The estimate of $(1-\beta)$ is 0.121 , with a t-statistic of 4.38 . The $\beta$ estimate implies a half-life of the deviations of 5.4 days, and 23 days to eliminate 95 percent of a deviation. Compared to MTAR, the TAR estimate of $\beta$ is larger, as expected, and the half-life and 95 percent return times are longer. The distribution of the Monte Carlo likelihood ratios and the maximized likelihood ratio for the TAR estimates were far above zero, confirming the results at the 95 percent confidence level.

Graph 3 shows the results for the crisis period. They are dominated by the two main periods of crisis. The Monte Carlo test did not confirm the TAR estimates for this period, that is the AR(1) restriction was not binding. The MTAR provides an estimate of the autoregression parameter in (13.2) for the domain A defined earlier in (12). This $\beta$ estimate is 0.90 with a t-statistic of 19.7 ; the t-statistic for $(1-\beta)$ is 2.2. This value of $\beta$ gives a half-life of 6.5 days, and a 95 percent recovery time of 27.9 days.

The results for the final period are shown in Graph 4. In the top panel we see a period of negative deviations from July 2000 - March 2001. The MTAR $\beta$ estimate is a high 0.91 , but it has a t-statistic for $(1-\beta)$ of 5.04. It has a half-life of 7.3 days and a 95 percent return time of 31.6 days. The $\beta$ estimate, half-life, and return time for TAR are 
even larger. The deviations in the top panel are small, but persistent, consistent with high estimates of $\beta$. The Monte Carlo test confirmed the TAR estimates. Thus during the tranquil period after the crisis, the deviations in the Hong Kong data are small, but they do follow an MTAR process. They are also persistent, with fairly high values for $\beta$.

The MTAR estimates are compared with TAR estimates for the same three periods in the second panels of Graphs 2 - 4. The comparisons use the MTAR estimate for the threshold, so the $\beta$ estimates are comparable. In the first and last periods where the TAR is confirmed by the Monte Carlo test, the MTAR provides a lower estimate of $\beta$ and, therefore, faster convergence. In the crisis period the thresholds are not significant, so the comparison is irrelevant. Thus the comparisons support the superiority of the MTAR model in the Hong Kong data.

\section{The Philippines}

The Philippine data are shown in Graph 5. These data show four distinct regimes of behaviour. The first is the period before the Asian crisis broke out, from August 1994 to June 1997. The second is the period of the crisis, from June 1997 to the end of April 1999. The third is the period from May 1999 to the end of August 2000. This is noisier than the first period. The fourth is the period from September 2000 to the end of March 2002. This seems to contain another crisis at the beginning, but possibly TAR behaviour after that.

The MTAR model has been estimated for each of these sub-periods, and the results are presented in Graphs 6 - 9, following the same format as the Hong Kong presentation. We begin the discussion of the results with Graph 6 for the first period. We will discuss these in some detail, and then summarize the rest of the results, since they follow the same format. The table in the second panel gives the results of the MTAR estimation for the period. An upper threshold of 0.017 percent is estimated; it can be located on the graph above. The estimate of $\beta$ is a high 0.935 , with a t-statistic of 40.96 . The t-statistic for $(1-\beta)$ is 2.84 , so $\beta$ is less than unity. The $\beta$ estimate gives a halflife of reversion of 10.3 days, with 44.6 days required to eliminate 95 percent of the deviation above the threshold. Thus the data in this period do follow an MTAR process, but with very slow reversion to the threshold. The Monte Carlo results did confirm the earlier estimates of the TAR model, so it appears that the MTAR performs better than the earlier TAR for this period. 
The results for the second period, beginning with the Asian crisis, are summarized in Graph 7. The Monte Carlo test did not confirm the earlier TAR estimates for this period. The MTAR estimates of the autoregression parameter $\beta$ in (13.2) in the domain $\mathrm{A}$ defined in (12) is 0.83 , with a t-statistic of 38.5 . The $\mathrm{t}$-statistic for $(1-\beta)$ is 7.18. This value of $\beta$ gives a half-life of 3.76 days and a 95 percent return time of 16.25 days.

The results for the third period are shown in Graph 8. There the threshold is 0.012 , with several episodes of deviation above it. The estimate of $\beta$ is 0.87 , with a $\mathrm{t}-$ statistic of 31.0. The t-statistic for $(1-\beta)$ is 4.62. The half-life is 5 days and the 95 percent return time is 21.6 days. The Monte Carlo test confirmed the earlier TAR estimates.

The results for the fourth period in Graph 9 have a threshold of 0.024, and a much lower $\beta$ of 0.63 . The t-statistic for $\beta$ is 11.2 , and the t-statistic for $(1-\beta)$ is 6.5. This value for $\beta$ gives a half-life of 1.5 days and a 95 percent return time of 6.55 days. The Monte Carlo test confirmed the earlier TAR estimates for this period.

The Philippine results did not confirm the TAR estimates for the second (crisis) period, as expected. The MTAR estimate of $\beta$ for the first period was a high 0.93 . The MTAR $\beta$ estimates for the third and fourth periods were 0.87 and 0.63 , respectively. These give more plausible return times. With shorter times in the later periods, it is likely that the market became more efficient over time in arbitraging deviations in the Philippine peso.

\section{Conclusion}

This paper has reported estimates of a TAR and a modified TAR model on the data for 3-6 month forward-forward deviations from CIP for the Hong Kong dollar and the Philippine peso on daily data from 1994 to 2002. The TAR model is confirmed by a Monte Carlo test for all parts of the data except the Asian crisis period of 1997-1999. The MTAR model provides lower estimates of the autoregression parameters and shorter return times in all cases where the TAR estimates were confirmed.

Earlier work on CIP deviations, for example Taylor and Peel (1998) and Tchernykh (1998), used the classical TAR model that was developed by Tong (1978). 
That model assumed that the CIP deviations appear as a leap outside a threshold, and then a regression back to the threshold. In the earlier review of the Asian data on CIP deviations [Tchernykh (2002b)] it was observed that deviations frequently develop as a series of jumps to a peak, and then the regression begins. Inclusion of these series of jumps in the estimation biases the estimate of the autoregression coefficient $\beta$ upward. To eliminate this bias, we developed the modified TAR (MTAR) model that eliminates the jump data from estimation of $\beta$. This model was used in estimation on the Hong Kong and the Philippine data.

The estimation of the TAR model on the Hong Kong and Philippine data was confirmed for periods before and after the Asian crisis. The MTAR and classical TAR were compared on the Hong Kong data, confirming the bias in the estimate of $\beta$ using the classical TAR model. The Philippine data are much noisier, and show a wider range of regimes. There the $\beta$ estimates for the non-crisis periods ranged from 0.93 to 0.62 . Thus the parameters of the MTAR models vary substantially across regimes. The MTAR for the Philippine data perform better the earlier TAR estimates.

The data on deviations from CIP in Hong Kong and the Philippines show large deviations before and during crises. Since these are 3-6 month forward-forward deviations, this implies that the spot-forward deviations get larger, the longer their maturities. This suggests that deviations from CIP, and estimates of the MTAR model, can be useful to policy-makers. These can be used by policy-makers as signals of bubbles and coming crises. These signals can lead policy-makers to inject liquidity into the market to prevent a coming crisis, or to allow the exchange rate to move as underlying policy changes are undertaken. They can be used by traders as the basis for trading rules for arbitrage when they have sufficient liquidity and secure counterparties. Both of these activities should work to close the deviations. 


\section{References}

Branson, W.H. (1969), “The Minimum Covered Interest Differential Needed for International Arbitrage Activity,” Journal of Political Economy, 77: 1028-35.

Corsetti, G., Pesenti, P. and Roubini, N. (1999), "What Caused the Asian Currency and Financial Crisis? Part I: A Macroeconomic Overview," Japan and the World Economy, 11: 305-73.

Einzig, Paul (1937), The Theory of Forward Exchange, London: MacMillan.

Einzig, Paul (1961), A Dynamic Theory of Forward Exchange, London: MacMillan.

Frenkel, J.A. and Levich, R.M. (1975), “Covered Interest Arbitrage: Unexploited Profit?” Journal of Political Economy, 83: 325-38.

Granger, C.W.J. and Terasvirta, T. (1993), Modelling Non-Linear Economic Relationships, Oxford University Press.

Grossman, S. and Stiglitz, J.E. (1980), “On the Impossibility of Informationally Efficient Markets.” American Economic Review, 70(3), 393-408.

Keynes, J.M. (1923), A Tract on Monetary Reform, London: Macmillan.

Taylor, Mark P. and Peel, David A. (1998), "Covered Interest Rate Parity in the InterWar Period and the Keynes-Einzig Conjecture," Working Paper, Department of Economics, Oxford University.

Taylor, Mark P. and Tchernykh, E. (2004), "Asymmetric Arbitrage and Default Premiums Between the U.S. and Russian Financial Markets," IMF Staff Papers, 51: 257-75. 
Tchernykh, E. (1998), "Estimation of CIP for Emerging Markets with an Application to Russia," Imperial College of Science, Technology and Medicine, the Management School.

Tchernykh, E. (2002a), “Testing Deviations from CIP on Brazilian Data,” Working Paper, Central Bank of Brazil.

Tchernykh, E. (2002b), "Testing the TAR Model and Deviations from Covered Interest Parity (CIP) along the Term Structure of Forward Markets," Working Paper, Hong Kong Institute for Monetary Research.

Tchernykh , E. (2004), “Application of a Modified TAR Model to CIP deviations in Asian Data”, Working paper No.19/2004, Hong Kong Institute for Monetary Research.

Tong, H. (1978), On a Threshold Model: In Pattern Recognition and Signal Processing, Sijthoff and Noordhoff, Amsterdam.

Tong, H. (1983), Threshold Models in Non-Linear Time Series Analysis, Lecture Notes in Statistics, No.21, Springer, Heidelberg. 


\section{Graph 1}

Hong Kong Foreign Exchange Forward-Forward Deviations.

These data were divided into 3 parts and the empirical analysis was done separately for each part.

\section{Forex Forward-Forward Deviations}

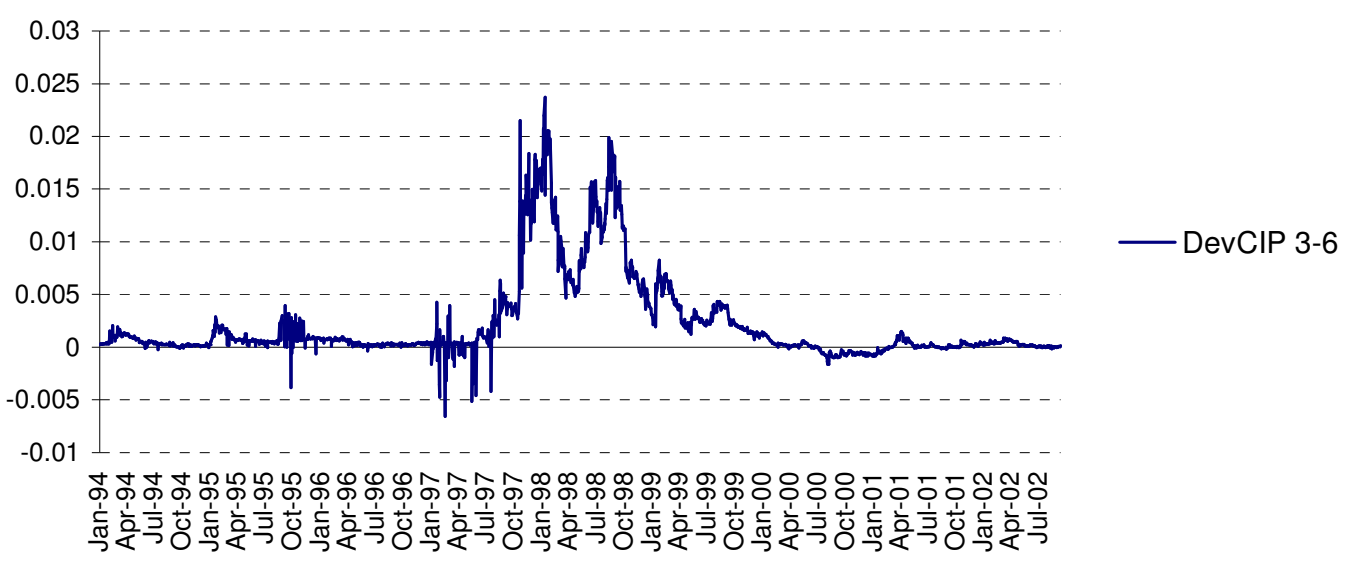


Graph 2

Part 1 Hong Kong

Forex Forward-Forward Deviations

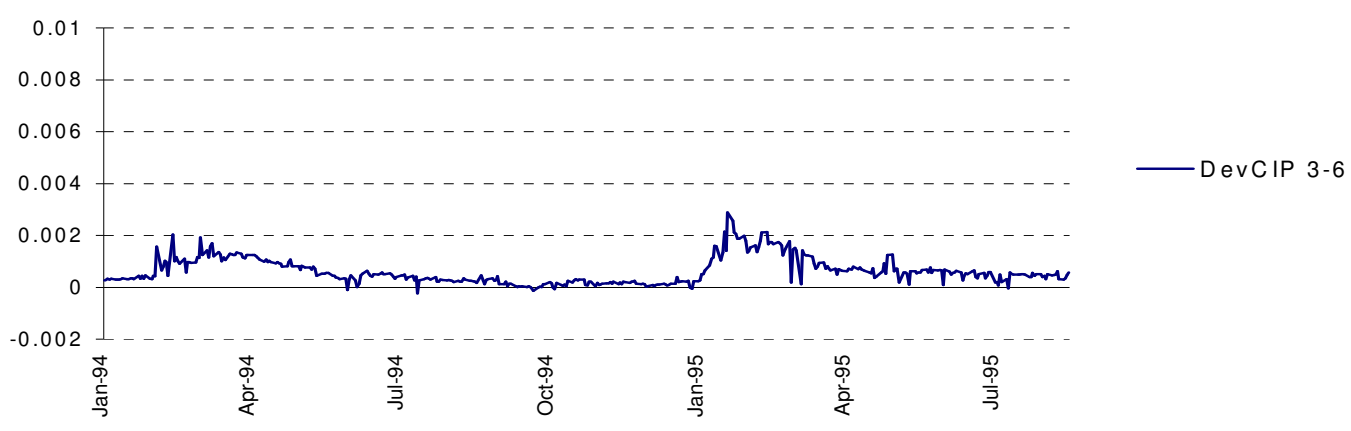

Part 1 Comparison, MTAR and TAR

(January 94 - August 95)

Hong Kong, Modified TAR

\begin{tabular}{lcccccr}
\hline \multicolumn{5}{c}{ Estimated Parameters BFGS-Method } \\
\hline Regimes & Thresholds & $\beta$ & T-Stat(0) & Half-Life & $\begin{array}{c}\text { 95\% Return } \\
\text { Time }\end{array}$ & T-Stat(1) \\
\hline Upper & 0.0007 & 0.8790204 & 31.754966 & 5.37543635 & 23.232249 & 4.3840 \\
Lower & None & & & & &
\end{tabular}

Hong Kong, TAR (unmodified)

\begin{tabular}{lcccccc}
\hline \multicolumn{7}{c}{ Estimated Parameters BFGS-Method } \\
\hline Regimes & Thresholds & $\beta$ & T-Stat(0)t & Half-Life & $\begin{array}{c}\text { 95\% Return } \\
\text { Time }\end{array}$ & T-Stat(1) \\
\hline Upper & 0.0007 & 0.9082582 & 24.618555 & 7.20328179 & 31.132066 & 2.4857 \\
Lower & None & & & & &
\end{tabular}


Graph 3

Part 2 Hong Kong

\section{Forex Forward-Forward Deviations}

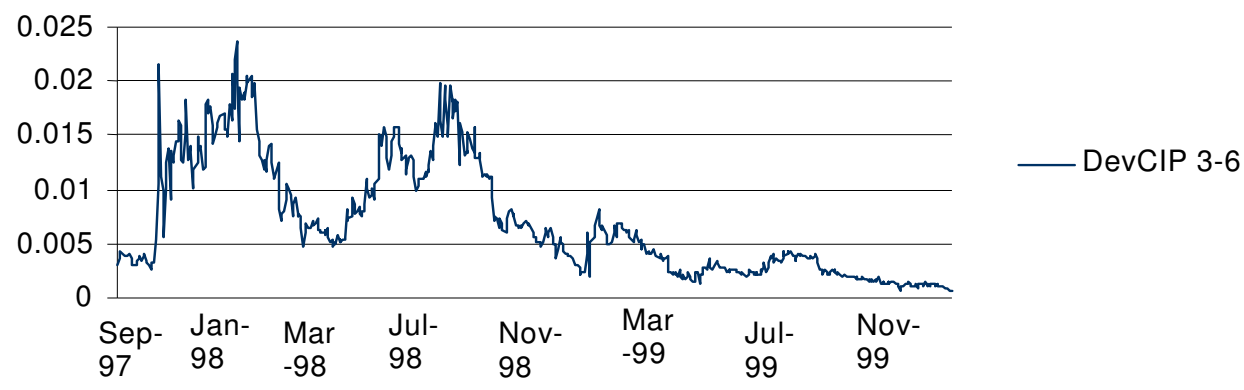

Part 2 TAR is rejected,

AR1 estimates of MTAR and TAR

(September 95 - April 2000, Crisis period)

Hong Kong, Modified TAR

\begin{tabular}{lcccccr}
\hline \multicolumn{7}{c}{ Estimated Parameters BFGS-Method } \\
\hline Regimes & Thresholds & $\beta$ & T-Stat & $\begin{array}{c}\text { Half-Life } \\
\text { Time }\end{array}$ & $\begin{array}{c}\text { 95\% Return } \\
\text { Time }\end{array}$ & T-Stat(1) \\
\hline Upper & 0.006 & 0.898338 & 19.671941 & 6.46539137 & 27.942957 & 2.2260 \\
Lower & None & & & & &
\end{tabular}

Hong Kong, TAR (unmodified)

\begin{tabular}{lcccccc}
\hline \multicolumn{6}{c}{ Estimated Parameters BFGS-Method } \\
\hline Regimes & Thresholds & $\beta$ & T-Stat & Half-Life & $\begin{array}{c}\text { 95\% Return } \\
\text { Time }\end{array}$ & T-Stat(1) \\
\hline Upper & 0.006 & 0.8841332 & 26.153292 & 5.62858981 & 24.32636 & 3.4269 \\
Lower & None & & & & &
\end{tabular}


Graph 4

Part 3 Hong Kong

Forex Forward-Forward Deviations

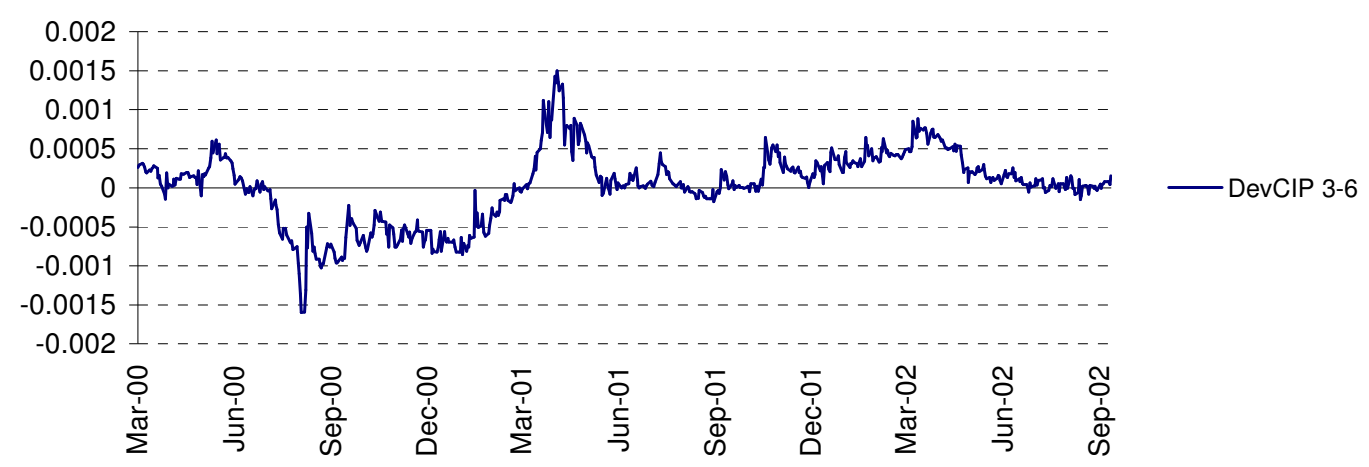

Part 3 Comparison, MTAR and TAR

(May 2000 - August 2002)

Hong Kong Modified TAR

\begin{tabular}{|c|c|c|c|c|c|c|}
\hline \multirow[b]{2}{*}{ Regimes } & \multicolumn{5}{|c|}{ Estimated Parameters BFGS-Method } & \multirow[b]{2}{*}{$T$-Stat(1) } \\
\hline & Thresholds & $\beta$ & T-Stat & $\begin{array}{c}\text { Half-Life } \\
\text { Time }\end{array}$ & $\begin{array}{c}95 \% \text { Return } \\
\text { Time } \\
\end{array}$ & \\
\hline $\begin{array}{l}\text { Upper } \\
\text { Lower }\end{array}$ & $\begin{array}{c}0.00005 \\
\text { None }\end{array}$ & 0.9096475 & 50.73474 & 7.31954561 & 31.63455 & 5.0391 \\
\hline
\end{tabular}

Hong Kong TAR (unmodified)

\begin{tabular}{lcccccr}
\hline \multicolumn{6}{c}{ Estimated Parameters } & BFGS-Method \\
\hline Regimes & Thresholds & $\beta$ & T-Stat & Half-Life & $\begin{array}{c}\text { 95\% Return } \\
\text { Time }\end{array}$ & T-Stat(1) \\
\hline Upper & 0.00005 & 0.9581789 & 60.126629 & 16.2250424 & 70.123467 & 2.6236 \\
Lower & None & & & & &
\end{tabular}




\section{Graph 5}

The Philippines Foreign Exchange Forward-Forward Deviations

These data were divided into 4 parts and the empirical analysis was done separately for each part.

\section{Forex Forward-Forward Deviations}

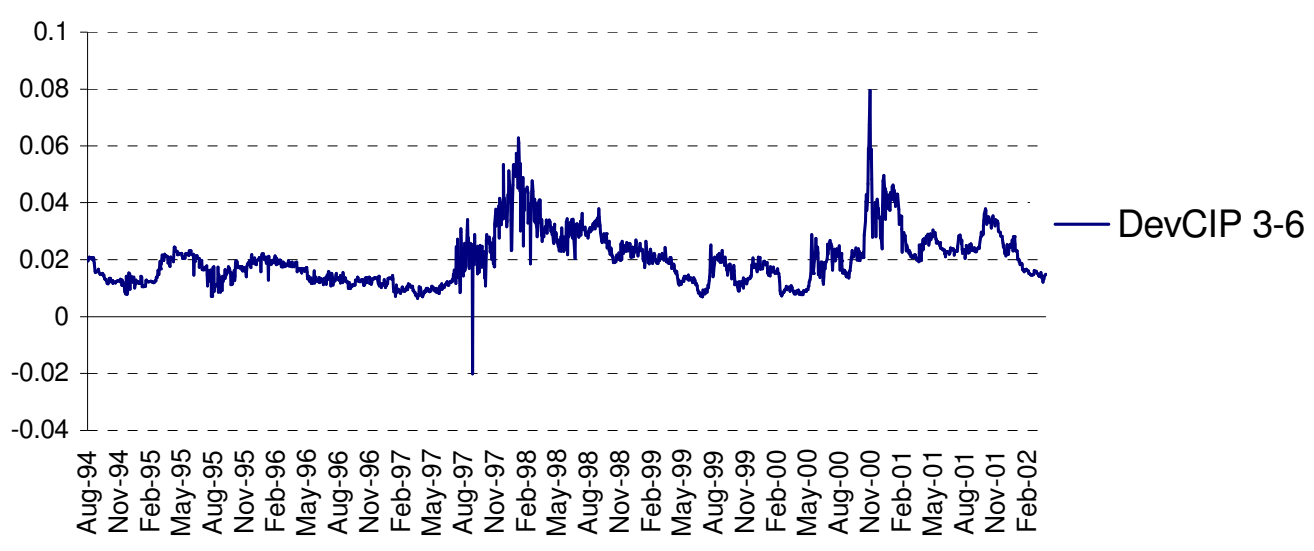




\section{Graph 6}

Part 1 The Philippines

August 94 - June 97

\section{Forex Forward-Forward Deviations}

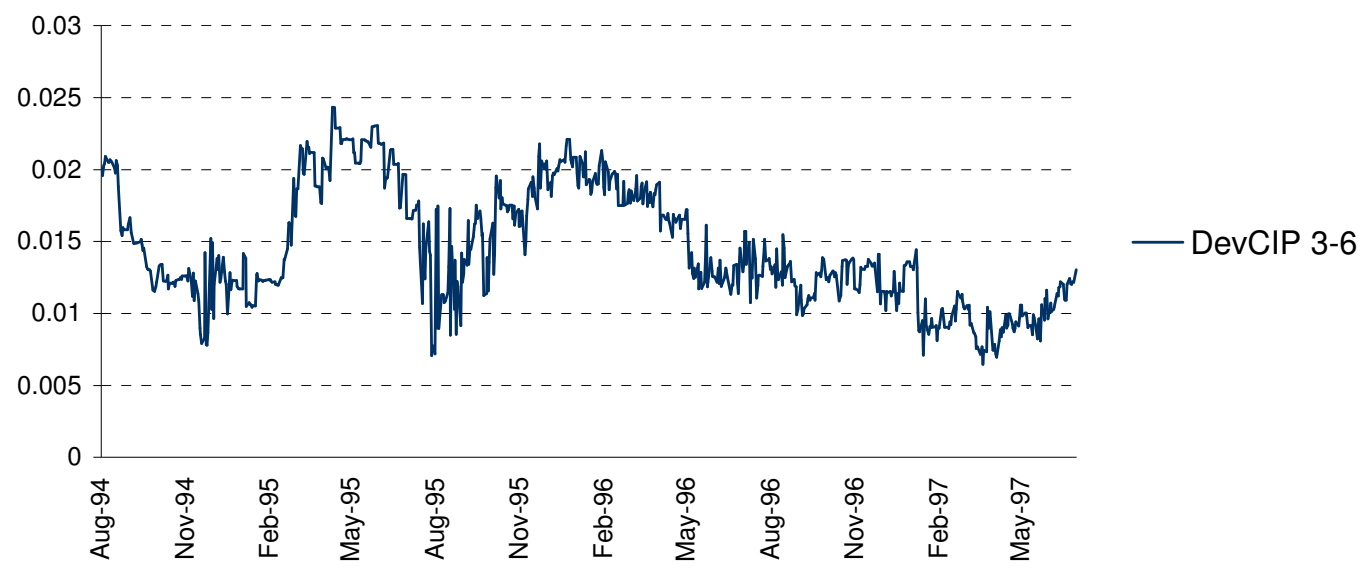

\section{MTAR}

\section{Estimated Parameters BFGS-Method}

\begin{tabular}{lcccccc}
\hline Regimes & Thresholds & $\beta$ & T-Stat(0) & Half-Life & $\begin{array}{c}\text { 95\% Return } \\
\text { Time }\end{array}$ & T-Stat(1) \\
\hline Upper & 0.017 & 0.9350798 & 40.956881 & 10.3264611 & 44.630222 & 2.8436 \\
Lower & None & & & & &
\end{tabular}




\section{Graph 7}

Part 2 The Philippines

July 97 - April 99, Crisis period

\section{Forex Forward-Forward Deviations}

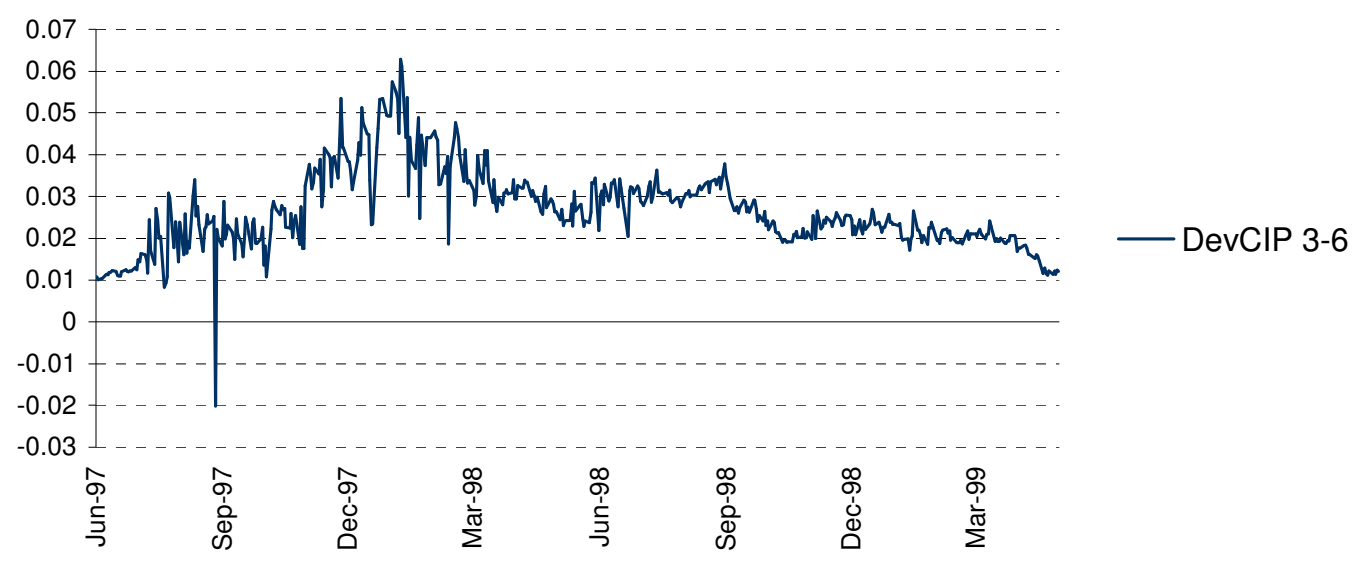

TAR is rejected, AR1 estimates of MTAR

Estimated Parameters BFGS-Method

\begin{tabular}{lcccccc}
\hline Regimes & Thresholds & $\beta$ & T-Stat(0) & Half-Life & 95\% Return & T-Stat(1) \\
& & & & Time & Time \\
\hline Upper & 0.025 & 0.8317062 & 35.485112 & 3.76146042 & 16.256761 & 7.1797 \\
Lower & None & & & & &
\end{tabular}




\section{Graph 8}

Part 3 The Philippines

May 99 - August 2000

\section{Forex Forward-Forward Deviations}

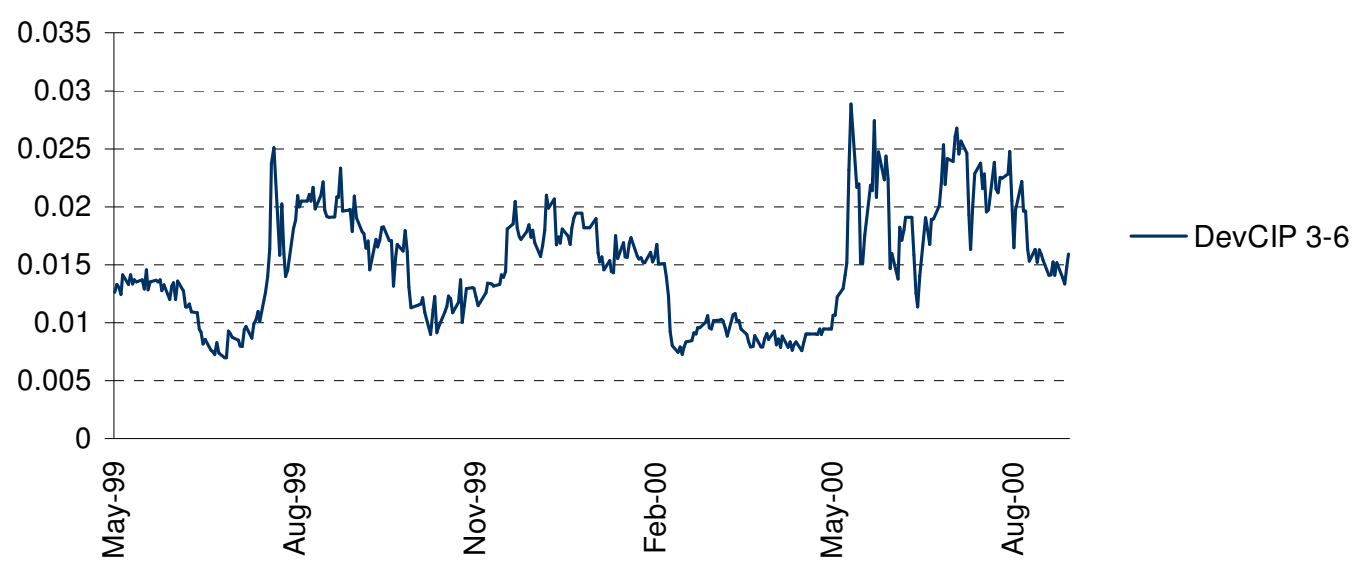

MTAR

\begin{tabular}{lcccccc}
\hline \multicolumn{7}{c}{ Estimated Parameters BFGS-Method } \\
\hline Regimes & Thresholds & $\beta$ & T-Stat(0) & Half-Life & $\begin{array}{c}\text { 95\% Return } \\
\text { Time }\end{array}$ & T-Stat(1) \\
\hline Upper & 0.012 & 0.8702267 & 31.004291 & 4.98661629 & 21.551797 & 4.6232 \\
Lower & None & & & & &
\end{tabular}




\section{Graph 9}

Part 4 The Philippines

September 2000 - April 2002

\section{Forex Forward-Forward Deviations}

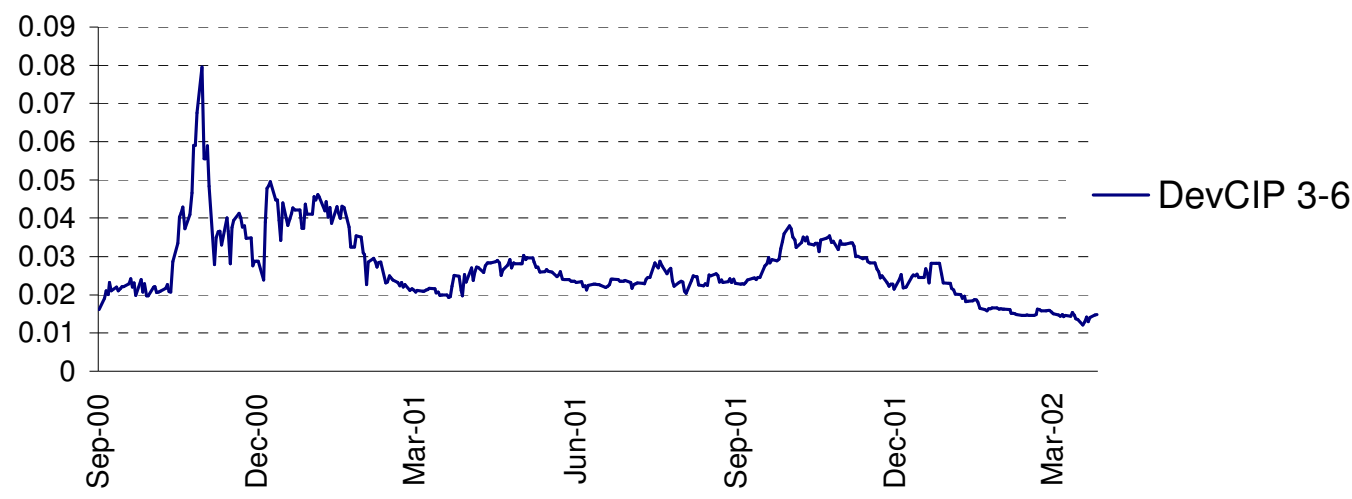

\section{MTAR}

\begin{tabular}{lcccccc}
\hline \multicolumn{6}{c}{ Estimated Parameters BFGS-Method } \\
\hline Regimes & Thresholds & $\beta$ & T-Stat(0) & Half-Life & $\begin{array}{c}\text { 95\% Return } \\
\text { Time }\end{array}$ & T-Stat(1) \\
\hline Upper & 0.024 & 0.6328683 & 11.212282 & 1.5150994 & 6.5481506 & 6.5048 \\
Lower & None & & & & &
\end{tabular}

